\title{
Rehabilitation Modality and Onset Differentially Influence Whisker Sensory Hypersensitivity after Diffuse Traumatic Brain Injury in the Rat
}

\author{
*Theresa Currier THOMAS ${ }^{1-3,6}$, *Ellen Magee STOCKHAUSEN ${ }^{5,6}$, L. Matthew LAW ${ }^{1,2}$, \\ Aida KHODADAD ${ }^{1,2}$, and Jonathan LIFSHITZ ${ }^{1-4,6}$ \\ *denotes that authors contributed equally to data collection, data interpretation, and \\ manuscript preparation
}

\footnotetext{
${ }^{1}$ Barrow Neurological Institute at Phoenix Children's Hospital - Phoenix, AZ ${ }^{2}$ Department of Child Health, University of Arizona College of Medicine - Phoenix, AZ ${ }^{3}$ Phoenix VA Healthcare System - Phoenix, AZ

${ }^{4}$ Neuroscience Program, Arizona State University - Tempe, AZ

${ }^{5}$ Core Medical Group, Manchester, NH

${ }^{6}$ Spinal Cord and Brain Injury Research Center, University of Kentucky Chandler Medical Center - Lexington, KY
}

Running Title: $\quad$ Rehabilitation Modality and Timing on Morbidity

\section{Corresponding Author:}

Theresa Currier Thomas, PhD Assistant Professor

Translational Neurotrauma Research Program

Department of Child Health, University of Arizona, College of Medicine - Phoenix Barrow Neurological Institute at Phoenix Children's Hospital 425 N. $5^{\text {th }}$ St.; $3^{\text {rd }}$ floor ABC-1 Building Phoenix, Arizona 85004 theresathomas@email.arizona.edu (602) 827-2505 (Office) (602) 827-2257 (Fax) Alternate email: tdcurr00@gmail.com 


\begin{abstract}
Background: As rehabilitation strategies advance as therapeutic interventions, the modality and onset of rehabilitation after traumatic brain injury (TBI) are critical to optimize treatment. Our laboratory has detected and characterized a late-onset, longlasting sensory hypersensitivity to whisker stimulation in diffuse brain-injured rats; a deficit that is comparable to visual or auditory sensory hypersensitivity in humans with an acquired brain injury.
\end{abstract}

Objective: We hypothesize that the modality and onset of rehabilitation therapies will differentially influence sensory hypersensitivity in response to the Whisker Nuisance Task (WNT) as well as WNT-induced corticosterone (CORT) stress response in diffuse brain-injured rats and shams.

Methods: After midline fluid percussion brain injury (FPI) or sham surgery, rats were assigned to one of four rehabilitative interventions: (1) whisker sensory deprivation during week one or (2) week two or (3) whisker stimulation during week one or (4) week two. At 28 days following FPI and sham procedures, sensory hypersensitivity was assessed using the WNT. Plasma CORT was evaluated immediately following the WNT (aggravated levels) and prior to the pre-determined endpoint 24 hours later (nonaggravated levels).

Results: Deprivation therapy during week two elicited significantly greater sensory hypersensitivity to the WNT compared to week one $(p<0.05)$, and aggravated CORT levels in FPI rats were significantly lower than sham levels. Stimulation therapy during week one resulted in low levels of sensory hypersensitivity to the WNT, similar to deprivation therapy and naïve controls, however, non-aggravated CORT levels in FPI rats were significantly higher than sham.

Conclusion: These data indicate that modality and onset of sensory rehabilitation can differentially influence FPI and sham rats, having a lasting impact on behavioral and stress responses to the WNT, emphasizing the necessity for continued evaluation of modality and onset of rehabilitation after TBI.

Key Words: rehabilitation, physical therapy, diffuse traumatic brain injury, whisker barrel circuit, sensory sensitivity 


\section{Introduction}

Traumatic brain injury (TBI) occurs as an instantaneous event, which results in lingering symptoms potentially leading to long-lasting neurological dysfunction (Masel \& DeWitt; Wood \& Rutterford, 2006). The yearly incidence of TBI is 2.5 million, accumulating a current estimation of 2.5-6.5 million survivors of TBI who live with residual disability (Coronado et al., 2011; O'Connor, Smyth, \& Gilchrist, 2011). Seventyfive percent of reported TBIs can be categorized as mild TBI or concussion (Control, 2003; Grossman, Inglese, \& Bammer, 2010); a brain injury associated with accelerationdeceleration of the brain inside the skull (O'Connor et al., 2011). Primary damage consists of diffuse axonal disruption that then leads to delayed neural degeneration and plasticity (Guimaraes et al., 2009; O'Connor et al., 2011). Common deficits include a reduction or impairment in cognitive function (e.g., memory and attention) (Ponsford, Cameron, Fitzgerald, Grant, \& Mikocka-Walus, 2011); motor control of posture (Sosnoff, Broglio, Shin, \& Ferrara, 2011), balance (Rinne et al., 2006; Sheedy, Geffen, Donnelly, \& Faux, 2006); and sensory processing such as auditory (Tapper, Gonzalez, Roy, \& Niechwiej-Szwedo, 2016), visual (Lew et al., 2011) and sensorimotor integration (Hall \& Lifshitz, 2010b; Lima et al., 2011; Niechwiej-Szwedo et al., 2007; Wilson, Powell, Gorham, \& Childers, 2006).

For sports concussion, cognitive rest is the primary recommendation (Eastman \& Chang, 2015). Cognitive rest implies limited driving of vehicles, television, video-games, use of computers, smart phones and tablets, socializing, school, homework, tests, exercising, and reading (Eastman \& Chang, 2015; Varner et al., 2017), thereby limiting not only cognitive processing, but sensory processing as well. However, alternate recommendations for recovery from TBI include light active rehabilitation, including talking, reading, playing games, and short-term computer use (Eastman \& Chang, 2015). Notably, the long-term effects of rest or light rehabilitation after diagnosis lack high quality evidence for either approach (Eastman \& Chang, 2015; Sawyer, Vesci, \& McLeod, 2016).

In rodent models of $\mathrm{TB}$, sensory deprivation may provide benefit by establishing a window of time for neural recovery to occur unguided by activity or sensory integration. Onset of intervention is also relevant to consider, as acute physical activity may have a 
negative effect on plasticity during week one after brain injury (Griesbach, GomezPinilla, \& Hovda, 2004). To evaluate onset and duration in addition to a task-specific nature, Matter et al., (2011) exposed brain-injured rats to either early or delayed environmental enrichment (EE) or standard housing interventions and evaluated motor and cognitive function. Significant motor recovery was evident in animals receiving early intervention, while cognitive tasks improved with delayed intervention indicating differential time windows for successful rehabilitation(Griesbach, Hovda, Tio, \& Taylor, 2011; Matter, Folweiler, Curatolo, \& Kline, 2011). To date, a clear time window for intervention has not been identified.

Laboratory guided neural plasticity following brain injury has been studied through activity interventions such as enriched environments (Gomez-Pinilla, Ying, Agoncillo, \& Frostig, 2011; Sozda et al., 2010) or exercise (Griesbach, Gomez-Pinilla, \& Hovda, 2007). Enriched environments (EE) have been used as a means of providing social, cognitive and sensory stimulation to guide neural recovery by inducing functional plasticity following brain injury (Gomez-Pinilla et al., 2011; Sozda et al., 2010). Exercise promotes neurogenesis, neuronal survival and neuroplasticity improving behavioral outcome and cognition in animal models, although consideration for timing, duration, intensity, or type of rehabilitation have not been critically assessed (Gomez-Pinilla, 2008; Griesbach, Tio, Vincelli, McArthur, \& Taylor, 2012; Wogensen, Mala, \& Mogensen, 2015). There is also conflicting evidence that premature exercise following TBI may actually deter neural plasticity (Griesbach, Gomez-Pinilla, et al., 2004).

In our laboratory, midline fluid percussion injury (FPI) induces a rat model of diffuse TBI that has been extensively studied to identify behavior, neurochemistry, inflammation and histopathology. FPI is well recognized as a consistent means to induce experimental diffuse brain injury(Cao, Thomas, Ziebell, Pauly, \& Lifshitz, 2012; Fenn et al., 2015; Griesbach et al., 2011; Hall \& Lifshitz, 2010a; Harrison et al., 2015; Hosseini \& Lifshitz, 2009; Learoyd \& Lifshitz, 2012; Lifshitz, Kelley, \& Povlishock, 2007b; Lifshitz \& Lisembee, 2012; Lifshitz et al., 2016; McNamara, Lisembee, \& Lifshitz, 2010; Thomas, Hinzman, Gerhardt, \& Lifshitz, 2012; Thomas et al., 2016; Thompson et al., 2005; Witgen et al., 2005) causing diffuse axonal injury similar to the pathology observed in human post-mortem brains (O'Connor et al., 2011). Accumulating data 
support a role for circuit reorganization in the late-onset, persisting TBI-induced sensory hypersensitivity to whisker stimulation. Specifically, the whisker system has been exploited as a somatosensory system that parallels the actions of the human cranial nerve V, the trigeminal nerve (Ciricillo et al., 1994; Sanders, Dietrich, \& Green, 2001). It is particularly useful as it controls the rat's primary sensory system which has a recognized motor component behavior pattern (Johansson \& Arvidsson, 1994) and resembles human sensorimotor integration behaviors such as in hand object manipulation or walking. The whisker barrel circuit effectively acts as the proprioceptive relay projecting information into the trigeminal ganglia through the ventral posterior medial nucleus of the thalamus (VPM) to the primary somatosensory cortex (S1BF) in organized columns, or whisker barrels (Ciricillo et al., 1994; Johansson \& Arvidsson, 1994; McNamara et al., 2010; Sanders et al., 2001). Using this model of injury-induced late-onset sensory hypersensitivity, experiments can be designed to test the impact of modality and onset of rehabilitation on injured and sham rats.

Late-onset sensory hypersensitivity to whisker stimulation was identified and characterized by our lab as the behavioral procedure termed the Whisker Nuisance Task (WNT)(McNamara et al., 2010). The WNT grades the behavioral responses of the rat to continuous stroking of the whiskers using a scoring paradigm to analyze eight distinct behaviors (McNamara et al., 2010). Results from this task have found that postTBI, rats demonstrate behaviors representative of fear, agitation, or avoidance, which are indicative of a sensory hypersensitivity associated with pathophysiology in the whisker barrel circuitry. Sham rats and rats at seven days post-injury respond with curious, relaxed behavior and normal whisking, confirming the hypersensitivity is not a continuous morbidity. The WNT detects whisker hypersensitivity at 28 days post-injury, thereby confirming WNT utility (McNamara et al., 2010).

In addition to behavioral outcomes, the WNT increases circulating levels of corticosterone (CORT) as an indication of injury and severity (McNamara et al., 2010). This is interpreted as a stress response to the normally non-noxious whisker stimulation, similar to a milder form of restraint stress (Griesbach et al., 2011; Rowe et al., 2016). In humans and rats, regulation of stress hormones are necessary for optimal brain and body functioning (Saavedra, Sanchez-Lemus, \& Benicky, 2011), as well as 
recovery from TBI (Gomez-Pinilla, 2008; Griesbach, Tio, Nair, \& Hovda, 2014; Kaplan, Vasterling, \& Vedak, 2010; Schaaf, De Kloet, \& Vreugdenhil, 2000; White-Gbadebo \& Hamm, 1993). Additionally, successful management of stress in TBI patients is linked to successful outcomes upon returning to home and community reintegration following TBI (Nalder et al., 2011). Therefore, successful management of post-TBI stress is a critical predictor of successful rehabilitation and may also be responsive to rehabilitation modality and onset.

In this study, we verified circuit-directed activation after stimulation therapy, using the immediate early gene (IEG) coding for activity regulated cytoskeleton associated protein (ARC; a.k.a. Arg3.1). ARC expression is a molecular marker of circuit activation that is specific, measurable, and input-dependent to the whisker circuit and has also been identified to play a role in plasticity (Khodadad, Adelson, Lifshitz, \& Thomas, 2015; Lyford et al., 1995). We then assessed whether modality and onset of rehabilitation after TBI differentially influences late-onset sensory hypersensitivity and consequential stress response using the WNT. To accomplish this we used two treatment paradigms: 1) deprivation treatment whereby the whiskers were shaved to prevent stimulation or 2) stimulation treatment whereby the rats were required to navigate plastic tubes to engage whisker activity in a functional environment in attempt to mitigate diffuse brain injury-induced late-onset sensory hypersensitivity. CORT levels were measured to determine if either rehabilitation modality or onset led to a reduction in whisker hypersensitivity induced stress. We propose that the success of either intervention is dependent on the timeframe of treatment initiation following TBI, divided into an early (week one) or late (week two) intervention period (see figure 1). These opposing approaches to intervention of whisker hypersensitivity reproduce an outpatient physical therapy setting, since the diffuse brain-injured rats are independent, mobile, feeding and grooming. 


\section{Materials and Methods}

\subsection{Midline Fluid Percussion Brain Injury}

Adult male Sprague-Dawley rats (350-375g; Harlan, Indianapolis, IN) were housed two per cage in a 12-h light/dark cycle with ad libitum access to food and water according to the Association for Assessment and Accreditation of Laboratory Animal Care International. Rats were acclimated to their environment for at least one week prior to experiments. After surgical procedures, each rat was monitored daily for postoperative health up to 5 days after surgery. The animal care techniques used were approved by the University of Kentucky Institutional Animal Care and Use Committee.

Rats were subjected to midline fluid percussion injury (FPI) consistent with methods described previously (Hoffman et al., 2016; Hosseini \& Lifshitz, 2009; Lifshitz, Kelley, \& Povlishock, 2007a; McNamara et al., 2010). Briefly, rats were anesthetized with $5 \%$ isoflurane in $100 \% \mathrm{O}_{2}$, placed in a stereotaxic frame and maintained with continuous $2 \%$ isoflurane throughout surgery. During surgery, body temperature was maintained with a Deltaphase ${ }^{\circledR}$ isothermal heating pad (Braintree Scientific Inc., Braintree, MA). In a head holder assembly (Kopf Instrument, Tujunga, CA), a midline scalp incision exposed the skull. A 4.8-mm circular craniectomy was performed (centered on the sagittal suture midway between bregma and lambda) without disrupting the underlying dura or superior sagittal sinus. An injury cap was fabricated from the female portion of a Luer-Loc needle hub, which was cut, beveled, and scored to fit within the craniectomy. A skull screw was secured in a 1-mm hand-drilled hole into the right frontal bone. The injury hub was affixed over the craniectomy using cyanoacrylate gel and methyl-methacrylate (Hygenic Corp., Akron, $\mathrm{OH}$ ). The incision was sutured at the anterior and posterior edges and topical Lidocaine ointment was applied. Rats were returned to a holding cage and monitored until ambulatory.

For injury induction, rats were re-anesthetized with 5\% isoflurane 60-90 min after surgery. The dura was inspected through the injury-hub assembly, which was then filled with normal saline and attached to the male end of the fluid percussion device (Custom Design and Fabrication, Virginia Commonwealth University, Richmond, VA). An injury of was administered by releasing the pendulum onto the fluid-filled cylinder as reflexive responses returned (1.9-2.0 atm). FPI rats were monitored for the presence of a 
forearm fencing response and the return of the righting reflex as indicators of injury severity (Hosseini \& Lifshitz, 2009; Learoyd \& Lifshitz, 2012; McNamara et al., 2010; Thomas et al., 2012). After injury, the injury hub assembly was removed en bloc, integrity of the dura was observed and the incision was stapled. Shams underwent the same surgical procedure without induction of injury. All FPI rats had righting reflexes between four minutes and nine minutes $(n=40)$. Sham rats had righting reflexes less than 15 seconds $(n=30)$. After recovery of the righting reflex, rats were placed in a holding cage until ambulatory before being returned to the vivarium. One FPI rat was euthanized due to excessive weight loss indicating unnecessary distress (occurred in $<3 \%$ of injured rats). Throughout this text, when stating 'time post-injury', both FPI and sham controls are included in the terminology unless otherwise stated.

\subsection{Whisker stimulation modalities}

Rats were assigned to one of four interventions (figure 1):

(1) Whisker deprivation (shaved whiskers) during week one post-injury;

(2) Whisker deprivation (shaved whiskers) during week two post-injury;

(3) Whisker stimulation (running through tubes) during week one post-injury; or

(4) Whisker stimulation (running through tubes) during week two post-injury.

Each intervention group contained two sets of rats; FPI and sham (described in section 2.1). A set of control rats that had sham surgery and no rehabilitation were included as reference parameters for comparison of modality and timing of rehabilitation therapy. The rats received a diffuse TBI using midline FPI (or sham) on day 0 . Rats were tested using the WNT on day 28, and underwent perfusion on day 29 (figure 1). Blood samples were taken immediately following WNT and prior to perfusion to evaluate 
plasma CORT as an indicator of stress. CORT samples taken after the WNT are termed 'aggravated' and those taken prior to perfusion are termed 'non-aggravated'.

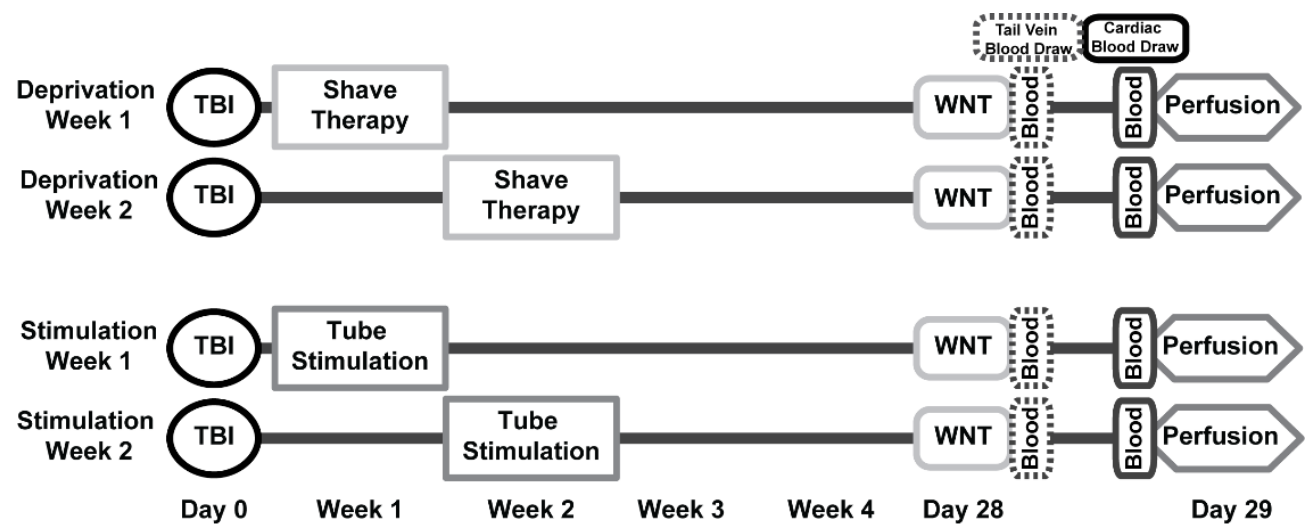

Figure 1. Schematic of the experimental design. Adult male Sprague-Dawley rats were assigned to one of four treatment groups. Each group received a diffuse midline fluid percussion injury or sham surgery on day 0 (TBI) and was tested for sensory hypersensitivity using the Whisker Nuisance Task (WNT) on day 28. In the deprivation treatment groups, mystacial whiskers were shaved to deprive sensory input during week one or week two post-injury. In the stimulation groups, rats performed trials through four tube configurations (Fig. 2), requiring them to use their whiskers to navigate the novel environment during week one or week two post-injury. Blood samples were collected twice during the study. Tail vein blood samples were taken from all rats immediately following the WNT and cardiac samples were acquired immediately prior to perfusion on day 29.

\section{3 "Deprivation" Intervention}

During week one or two post-injury, rats received deprivation intervention by having their whiskers shaved to minimize somatosensory circuit activation to simulate mandatory 'rest' that is often recommended after concussion. Whiskers were shaved close to the mystacial pads using an electric razor while lightly restraining the rat. In "deprivation week 1" (sham: $n=6$; FPI: $n=9$ ), the whiskers were shaved on the first day post-injury and again four days later; in "deprivation week two" (sham: $n=6 ;$ FPI: $n$ $=10$ ), the whiskers were shaved on the eighth day post-injury and repeated four days later. Rats received no other intervention until administration of the WNT at 28 days post-injury.

\subsection{Whisker "Stimulation"}

Two other groups of rats underwent whisker stimulation intervention to stimulate the somatosensory whisker circuit. Stimulation therapy involved each rat navigating through four different configurations of opaque PVC tubing $(122 \mathrm{~cm}$ in length, $7.5 \mathrm{~cm}$ in diameter, figure 2), from each direction, for 8 trials on one day (figure 2). Rats were 
exposed to stimulation therapy three times every other day during the week of intervention to simulate aspects of outpatient physical therapy environment (see discussion). "Stimulation week one" (sham: $n=6 ; F P I: ~ n=10)$ completed this intervention during the first week post-injury; "Stimulation week two" (sham: $n=6$; FPI: $n$ $=10$ ) completed this intervention during the second week post-injury. The PVC tubes were constructed from 4-5 pieces of plumbing pipe in the lab and were cleaned with Roccal-D Plus ${ }^{\circledR}$ (Pfizer, New York) prior to the first rat and between each rat. Rats received no other intervention until the WNT was conducted at 28 days.

Each rat was timed and subjectively scored on its performance through each tube. One minute was allowed for the rats to traverse the length of tube independently. For each trial, exploration was rated "fast" if the trial was completed in less than 30 seconds and "slow" if it took longer than 30 seconds. Emergence (exit) from the tube was scored as "easy" if rats were retrieved only by removing one piece of tubing and "hard" if rats back-tracked through the tube and required removal of more than one piece of tubing for retrieval. When rats did not complete the length of the tube within this time limit, they were gently motivated to complete the trial with a large bottle brush, as to minimize or avoid a stress response. Individual trials for rats requiring motivation were marked as "motivated." For each rat, there were 8 total tube stimulation trials a day (4 tubes $\times 2$ directions) over three days for a total of 24 scored trials per rat. One point was scored for fast rather than slow, easy rather than difficult, or motivated rather than not motivated for each trial. A percentage was calculated for each rat every day and then averaged across days to be used for statistical analysis.

(\# fast, easy or motivated trials/total number of trials) * 100 

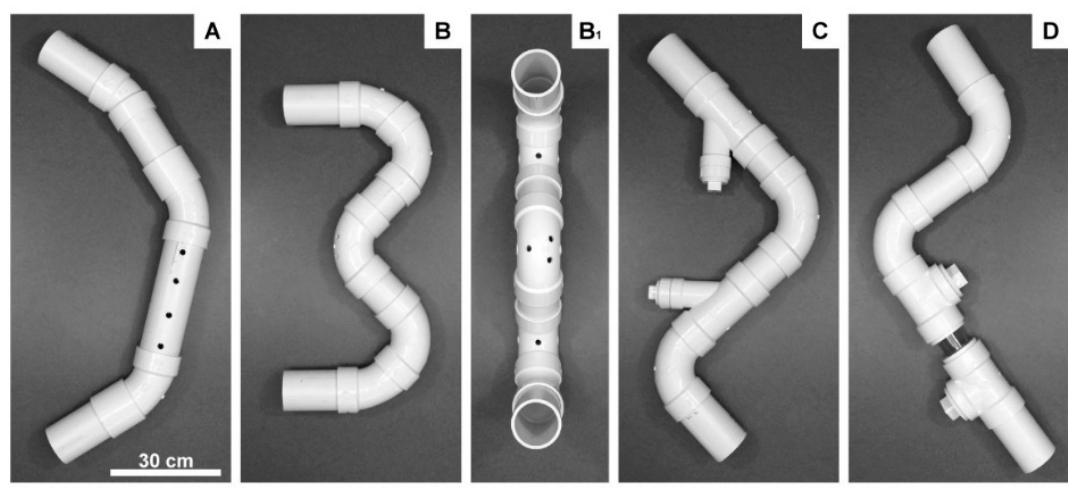

Figure 2. Tube configurations used for stimulation therapy. Four configurations $(A, B, C, D)$ of plumbing pipe were designed to provide whisker stimulation during tactile navigation as the rat navigated through the length of tubing. Rats assigned to tube stimulation therapy groups performed eight trials through the tubes, once through each tube starting from each end. Trials were performed three times per week for a single week; either during week one or week two post-injury. Note the variety of joints, turns, dead-ends and 1.5 $\mathrm{cm}$ holes along the lengths of the tubes to provide tactile variety. Tube B can be seen on edge (B1) to demonstrate the tube diameter and additional holes.

\subsection{Verification of Circuit-Directed Activation After Tube Stimulation Therapy}

Similar to section 2.4, each rat navigating through four different configurations of opaque PVC tubing, from each direction to avoid a learning effect, for a total of 8 navigation trials (figure 2). At 15, 30, 60, 180 minutes following the conclusion of stimulation therapy ( $n=4 /$ time point), brains were harvested. Naïve rats were brought directly from their holding room and their brains harvested as non-stimulated controls $(n=4)$. Biopsies from two of the whisker barrel circuit relays, the VPM and S1BF, were stored in RNAlater ${ }^{\circledR}$ at $-20^{\circ} \mathrm{C}$ until RNA was extracted.

RNA was extracted using the MagMAX ${ }^{\mathrm{TM}}$-96 Total RNA Isolation Kit (Invitrogen catalog; \# AM1830). Following the manufacturer's protocol, biopsies were homogenized in TRI reagent ${ }^{\circledR}$ solution (Invitrogen; \# AM9738) and extracted under acidic conditions.

Total RNA was further purified using RNA binding beads. The ratio of the absorbance at 260 and $280 \mathrm{~nm}$ was used to assess RNA purity and quality (Nanodrop, ThermoScientific 2000). All RNA samples were within the established range for pure RNA (1.82.1).

Total RNA was converted to cDNA using the High Capacity RNA-to-cDNA Kit from Life Technologies ${ }^{\mathrm{TM}}$ (catalog \# 4387406). Each sample of concentrated cDNA was diluted to $5 \mathrm{ng}$ in preparation for quantitative real-time PCR (qPCR) using Applied 
Biosystems TaqMan® Gene Expression Assay for ARC (Rn00571208_g1) which was optimized to run under universal thermal cycling conditions, with amplification efficiencies of $100 \%$ by the manufacturer. Within each rat, relative gene expression was normalized to the 18S rRNA endogenous control and the expression level in the sham/vehicle group using the $2^{-\triangle \Delta C T}$ method (Livak \& Schmittgen, 2001), which relates gene expression to the PCR cycle number at which the fluorescence signals exceed a threshold above baseline. Samples were run in triplicate and $18 \mathrm{~s}$ was run in duplicate according to manufacturer's instructions.

\subsection{Whisker Nuisance Task}

Behavioral responses to whisker stimulation were assessed using the WNT, as previously described (McNamara et al., 2010). At 28 days after FPI or sham surgery, rats were acclimated for 5 minutes to a plastic test cage $(57.1 \times 39.4 \times 15.2 \mathrm{~cm})$ lined with an absorbent pad. The whiskers of both mystacial pads were manually stimulated with a wooden applicator stick for three consecutive five minute periods (15 min total). Between periods, stimulation was absent while behavioral observations were recorded. The same observer, who was blinded to injury status, conducted behavioral testing.

Rat behaviors were analyzed during each 5-minute period of the session and rated in eight previously developed categories of behavioral expression. The predominant behavioral responses included: freezing, stance and body position, breathing, whisker position, whisking response, evasiveness, response to stick presentation, and grooming on 0-2 point non-parametric scales ( $0=$ absent, $1=$ present, $2=$ profound). Normal behavioral responses to stimulation were assigned a zero value, whereas meaningful abnormal behavioral responses were assigned a value of 2 . The maximum whisker nuisance score is 16. High scores (6+) indicate abnormal responses to the stimulation, in which the rat freezes, becomes agitated or is aggressive. Low scores (0-4) indicate normal responses, in which the rat is either curious or ambivalent to the stimulation (Learoyd \& Lifshitz, 2012; McNamara et al., 2010; Thomas et al., 2012). 


\subsection{Corticosterone Assay}

Blood samples were collected twice during the study, over fixed time periods in the afternoon. Tail vein blood samples were taken from all rats immediately following WNT on day 28 post-injury to evaluate plasma CORT levels when rats were aggravated. Rats were placed into a Flat Bottom Restrainer (Braintree Scientific, INC) and adjacent to two Deltaphase Isothermal Pads (Braintree Scientific, INC) to increase body temperature. Blood was drawn from a lateral tail vein using Terumo Surflo Winged Infusion Set 23GX3/4" from butterfly needles (Fisher Scientific) into a heparinized centrifuge tube. Rats were euthanized by an overdose of sodium pentobarbital (150 mg/kg, i.p.) and cardiac blood samples were taken prior to transcardial perfusion on day 29 post-injury to evaluate plasma CORT levels when rats were not aggravated.

All blood samples were centrifuged (3000g, $10 \mathrm{~min}$ ) and plasma stored at $-20^{\circ} \mathrm{C}$. A commercially available competitive immunoassay was conducted according to manufacturer's protocol for the quantitative determination of CORT (ELISA, Assay Designs, 900-097). The kit uses an anti-CORT polyclonal antibody to bind standards and samples. The enzyme reaction generates a yellow color that is inversely proportional to the CORT concentration and read on a microplate reader ( $405 \mathrm{~nm}$ ). All samples were diluted 1:5 (80\%) in order to stay within the sensitivity of the assay (32$20,000 \mathrm{pg} / \mathrm{mL})$.

\subsection{Data Analysis and Statistics}

The percent of fast travel, easy removal, and requirement of motivation during the stimulation therapy was analyzed using Chi-Square analysis. The average of the total whisker nuisance scores for each rat was incorporated into a Mann-Whitney unpaired, 2-tailed, non-parametric test that was used to determine statistical differences between therapeutic interventions (deprivation vs. stimulation), therapeutic efficacy of treatment timing (week one vs. week two) and rat groups (sham vs. FPI). Plasma CORT levels were evaluated based on therapeutic intervention (deprivation vs. stimulation), after the WNT or prior to perfusion (aggravated vs. non-aggravated), timing of therapy (week one vs. week two) and injury status (sham vs. FPI) using mixed ANOVAs. Bonferroni's posthoc comparisons were used for all parametric data. Statistical significance was 
considered at $p<0.05$. All experiments were carried out with investigator blinded to the injury status and therapy of the rat.

Rats were randomly assigned to one of four interventions using a random number generator. Investigators blind to injury status and rehabilitation intervention carried out behavioral tasks and blood collection. Inclusion criterion injured rats was a righting reflex time of 4-9 minutes. Exclusion criterion included weight loss greater than $10 \%$ of surgery weight (one rat; replaced with another rat), tail vein blood samples that were not acquired within seven minutes of being placed in the rodent restrainer (two rats), and blood samples that did not have both aggravated and non-aggravated CORT levels for comparisons using repeated measures (four rats). Of the six rats, one was a sham from deprivation week one, one was a sham from stimulation week one, one was injured from stimulation week one, two were shams from stimulation week two, and one was injured from deprivation week two. Two statistical outliers from the VPM data set for rtPCR were determined using the ROUT method, where q=1\% (Graphpad Prism7; La Jolla, CA).

\section{Results}

\subsection{Stimulation therapy results in activation of the whisker circuit}

At multiple time points after stimulation therapy, tissue biopsies from the VPM and S1BF relays of the whisker barrel circuit were processed for $\mathrm{QPCR}$ in comparison with naïve rats. Whisker stimulation resulting from stimulation therapy resulted in a 8-fold increase in ARC mRNA expression at 30 minutes in the S1BF in comparison to naive rats $(F(4,13)=4.42 ; p<0.05$; figure 3$)$. There were no changes in ARC expression in the VPM. These data support that stimulation therapy robustly and specifically activated the S1BF of whisker barrel circuit. 


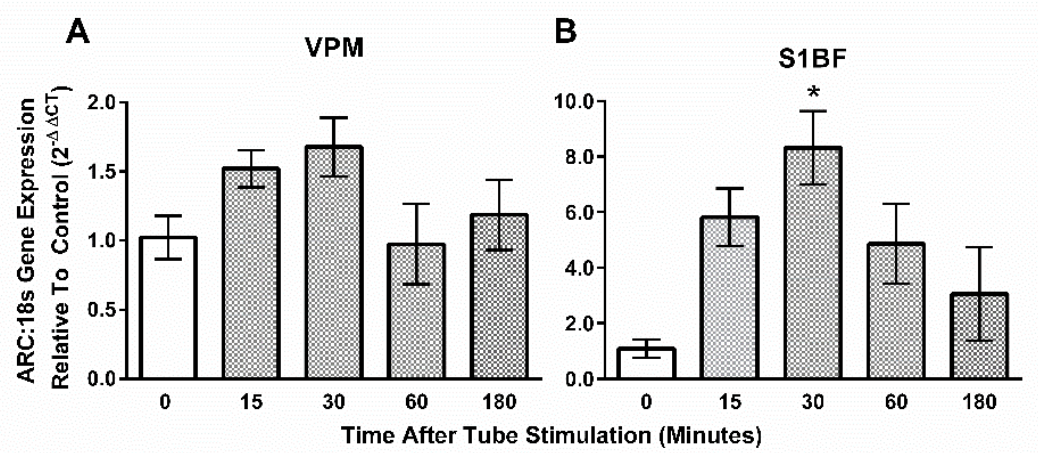

Figure 3. Tube stimulation therapy is capable of activating the whisker circuit. (A) No changes in ARC mRNA expression were detected in the VPM. Stimulation therapy resulted in an 8-fold increase of ARC mRNA expression at 30 minutes in the S1BF in comparison to naive. Bar graphs represent the mean \pm SEM. ${ }^{*} \mathrm{p}<0.05 ; \mathrm{n}=4$ /group.

\subsection{Performance on stimulation therapy}

Trials through each tube configuration (figure 2) were recorded and scored over the three days to determine the percentage of fast progression, easy removal, or trials requiring motivation in sham and injured $(\mathrm{FPI})$ rats during therapy at weeks one and two post-injury (Table 1). Fast progression, referring to the speed at which the rats navigated the tubes, changed as a function of therapy onset $(63.8 \% \pm 0.05 \mathrm{wk} 1 \mathrm{vs}$. $78.9 \% \pm 0.04 w k 2 ; U=72 ; p<0.05)$ but not as a function of injury status $(69.1 \% \pm 0.04$ all sham vs. $72.7 \% \pm 0.05$ all FPI; $U=98 ; p=0.39$ ). FPI rats were easier to remove from the tubes during week two therapy $(36.1 \% \pm 0.05$ sham vs. $61.0 \% \pm 0.04 \mathrm{FPI} ; U=5.0 ; p<0.05)$, however ease of removal was similar between sham and injured rats during week one $(\sim 47 \%)$ and an overall effect of therapy onset $(U=118 ; p=0.72)$ was not detected.

Motivation to navigate the tubes was not significantly changed as a function of therapy onset $(U=81 ; p=0.07)$ or injury status $(U=98 ; p=0.387)$. All rats successfully completed stimulation therapy. No differences in rat performance were detected during week one therapy after surgery; however, during week two therapy, all rats moved more quickly through the tubes and FPI rats were more easily removed from the tubes at the end of the trial. 


\begin{tabular}{lcccccc} 
& \multicolumn{2}{c}{ Week 1 } & \multicolumn{2}{c}{ Week 2 } \\
& Sham & FPI & Overall Wk1 & Sham & FPI & Overall Wk2 \\
Fast progression & $65.3 \% \pm 0.04$ & $62.9 \% \pm 0.08$ & $63.8 \% \pm 0.05$ & $72.9 \% \pm 0.08$ & $82.5 \% \pm 0.05$ & $78.9 \% \pm 0.04^{\#}$ \\
Easy removal from tube & $47.9 \% \pm 0.05$ & $47.3 \% \pm 0.06$ & $47.6 \% \pm 0.04$ & $36.1 \% \pm 0.05$ & $61.0 \% \pm 0.05^{*}$ & $51.7 \% \pm 0.05$ \\
Required motivation & $9.0 \% \pm 0.03$ & $27.1 \% \pm 0.07$ & $20.3 \% \pm 0.05$ & $11.8 \% \pm 0.05$ & $8.3 \% \pm 0.03$ & $9.6 \% \pm 0.03$
\end{tabular}

Table 1: Characteristic performance in the tube stimulation trials for uninjured sham ( $n=6 /$ group) and fluid percussion brain-injured ( $F P I)$ rats ( $n=10 /$ group). Values are mean \pm SEM for the percent of rats in each group that showed fast progression through the tubes, easy removal from the tube, and required motivation to complete the trial. ${ }^{*}, p<0.05$ compared to sham; ${ }^{*} p<0.05$ in comparison to week one.

\subsection{Deprivation therapy during week two increased sensory hypersensitivity Here, we investigated whether early deprivation or stimulation therapy could} influence sensory hypersensitivity to whisker stimulation at 28 days post-injury. There were no significant differences between WNT scores for sham $(n=6)$ and FPI $(n=10)$ rats for either therapy (deprivation $U=24.5 ; p=0.58$; stimulation $U=28 ; p=0.87$ ). However, there was an overall therapy effect for deprivation $(n=31)$ and stimulation $(n=32)$ treated rats, where a Mann-Whitney unpaired, 2-tailed, nonparametric comparison indicated that deprivation therapy treated rats had significantly higher WNT scores $(4.71 \pm 0.68$ and 1.46 \pm 0.41 , respectively; $U=187 ;{ }^{*} p<0.05$, figure 4 ). This suggests that at 28 days post-injury, rats undergoing deprivation therapy were significantly more sensitive to the WNT compared to rats receiving stimulation therapy. In addition to the sham controls, a group of naïve rats receiving no surgery and no therapeutic treatment were also subjected to the WNT at 28 days post-injury for a baseline comparison $(2.27 \pm 0.81 ; n=5$, represented as the dotted line in figure 4). Other comparisons will be discussed prior to the corresponding CORT levels below. 


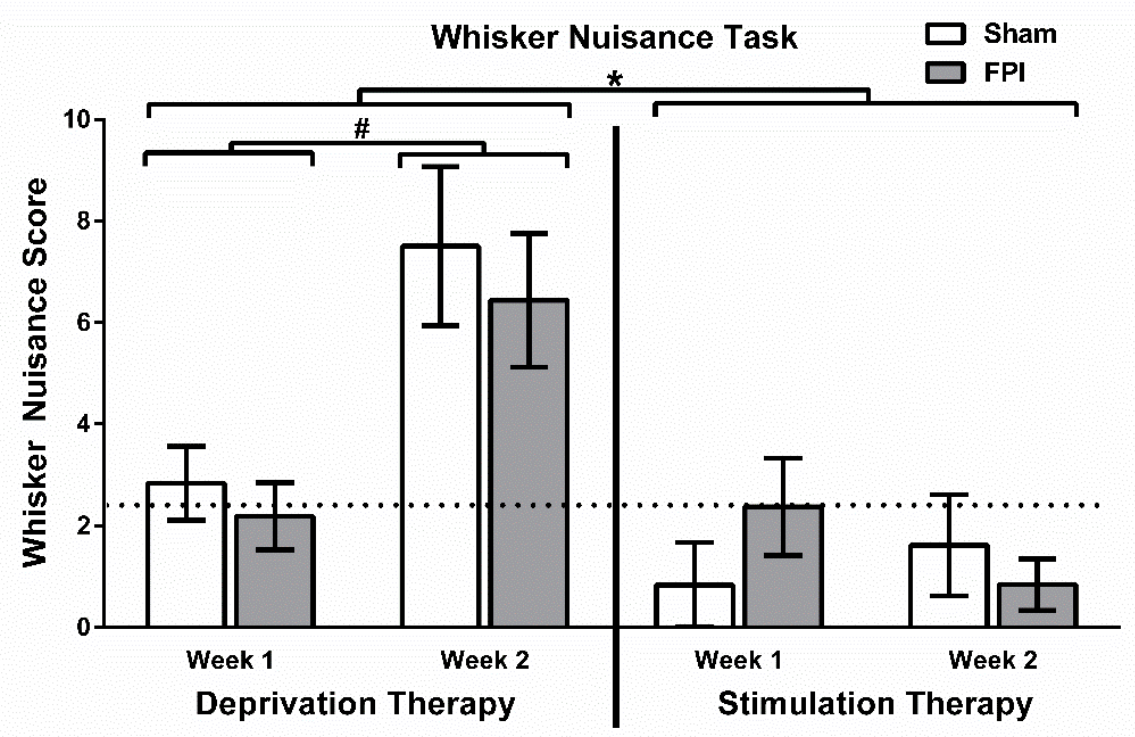

Figure 4. Does timing and modality of rehabilitation influence whisker nuisance task scores? Overall, deprivation therapy resulted in higher whisker nuisance scores than stimulation therapy $\left(^{*}\right)$, with deprivation therapy during week two post-injury being significantly higher than week one post-injury (\#). Deprivation therapy during week one and stimulation therapy during weeks one and two resulted in scores similar to naive controls (horizontal dotted line). Injury status did not reflect in whisker nuisance scores. Bar graphs represent the mean \pm SEM. ${ }^{*}, p<0.05$. Horizontal dotted line represents whisker nuisance scores of naive rats $(n=3)$.

\subsection{The effect of therapeutic intervention on CORT levels in response to the WNT}

Circulating levels of plasma CORT were measured as an indicator of the physiological stress to the WNT (aggravated CORT levels) and again at 24 hours later, prior to perfusion (non-aggravated). Based on our previous publication, injured rats have a greater CORT response to the WNT (McNamara et al., 2010). First, we compared the average of the aggravated and non-aggravated levels of CORT between the two therapies, regardless of injury or timing of therapy, with a repeated measures ANOVA. The results showed a main effect of therapy $\left(F(1,51)=18.11,{ }^{\$} p<0.05\right)$ indicating a significantly larger increase in CORT levels following deprivation compared to stimulation therapy (figure 5). There was also a main effect of overall aggravated vs non-aggravated CORT levels $(F(1,51)=13.74, p<0.05)$ indicating that the WNT increased CORT levels, regardless of therapy type, therapy onset or injury status (data further evaluated below; comparison not highlighted in figure). However, there was not a significant interaction $(F(1,51)=0.09, p=0.764)$ because CORT returned to nonstimulated levels within 24 hours (non-aggravated levels). In summary, when pooling 
week of therapy onset, aggravated+non-aggravated CORT levels, and sham+FPI rats to determine the overall effect of therapy on CORT levels, deprivation therapy resulted in higher CORT levels in comparison to stimulation therapy. When assessing nonaggravated vs. aggravated CORT levels regardless of therapy type, the WNT elevated CORT levels in both therapy groups.

Next, we combined all of the sham and FPI rats in the deprivation $(n=29)$ or stimulation ( $n=24)$ therapy groups, regardless of therapy onset, to assess whether deprivation or stimulation therapy influenced aggravated CORT levels in a between group comparison. The results of an unpaired t-test showed that rats with deprivation therapy had significantly greater aggravated CORT levels compared to aggravated CORT levels in the stimulation therapy group $(139.64 \pm 14.83 \mathrm{vs} 95.53 \pm 9.66 \mathrm{ng} / \mathrm{ml}$, respectively; $t(51)=2.37 ;{ }^{+} p<0.05$; figure 5 ). Non-aggravated CORT levels in the deprivation therapy group were significantly higher compared to the non-aggravated levels in the stimulation therapy group $(93.82 \pm 12.83 \mathrm{vs.} 41.61 \pm 9.48 \mathrm{ng} / \mathrm{ml}$, respectively; $t(51)=3.15$; ${ }^{*} p<0.05$; figure 5). These data indicate that both aggravated and nonaggravated CORT levels of deprivation therapy treated rats are elevated in comparison to aggravated and non-aggravated CORT levels of stimulation therapy rats.

Then we compared the aggravated and non-aggravated CORT levels within deprivation and stimulation therapies independently (FPI and sham still combined) using a paired t-test. For rats that received deprivation therapy, aggravated CORT levels following the WNT were significantly higher in comparison to non-aggravated levels $\left(139.64 \pm 14.83\right.$ vs. $93.82 \pm 12.83 \mathrm{ng} / \mathrm{ml} ; t(28)=2.19$, ${ }^{\circledR} p<0.05$; figure 5$)$. For rats with stimulation therapy, there is also a significant increase in CORT levels after the WNT that significantly decreases 24 hours following the WNT (95.53 \pm 9.66 vs. $41.61 \pm 9.48$ $\mathrm{ng} / \mathrm{ml} ; t(23)=3.50,{ }^{*} p<0.05$; figure 5$)$. This indicates that following whisker stimulation, rats receiving deprivation and stimulation therapy had higher levels of CORT in comparison to their non-aggravated levels, indicating that the high CORT levels were due to hypersensitivity to the WNT.

Averages are presented in reference to the naïve control CORT levels (blood sample taken prior to perfusion) as represented with a dotted line in the bar graphs 
$(60.09 \pm 20.02 \mathrm{ng} / \mathrm{ml} ; \mathrm{n}=3)$. It is noteworthy that non-aggravated levels of shams in the stimulation therapy group were significantly lower than naïve animals $(\mathrm{t}(6)=2.67$; $p<0.05$ ); such that, while WNT-induced significant increases in CORT levels, the aggravated CORT levels were similar to naïve $(t(7)=0.13 ; p=0.90)$. The results suggest that the WNT leads to elevated levels of CORT, regardless of the therapy, that are then reduced by 24 hours after whisker stimulation. Injury effects will be further discussed in figures 6 and 7.

Overall, the results suggest that regardless of rehabilitation modality, WNT caused an increase in CORT levels. However, CORT levels from deprivation therapy were higher than stimulation therapy on all accounts.

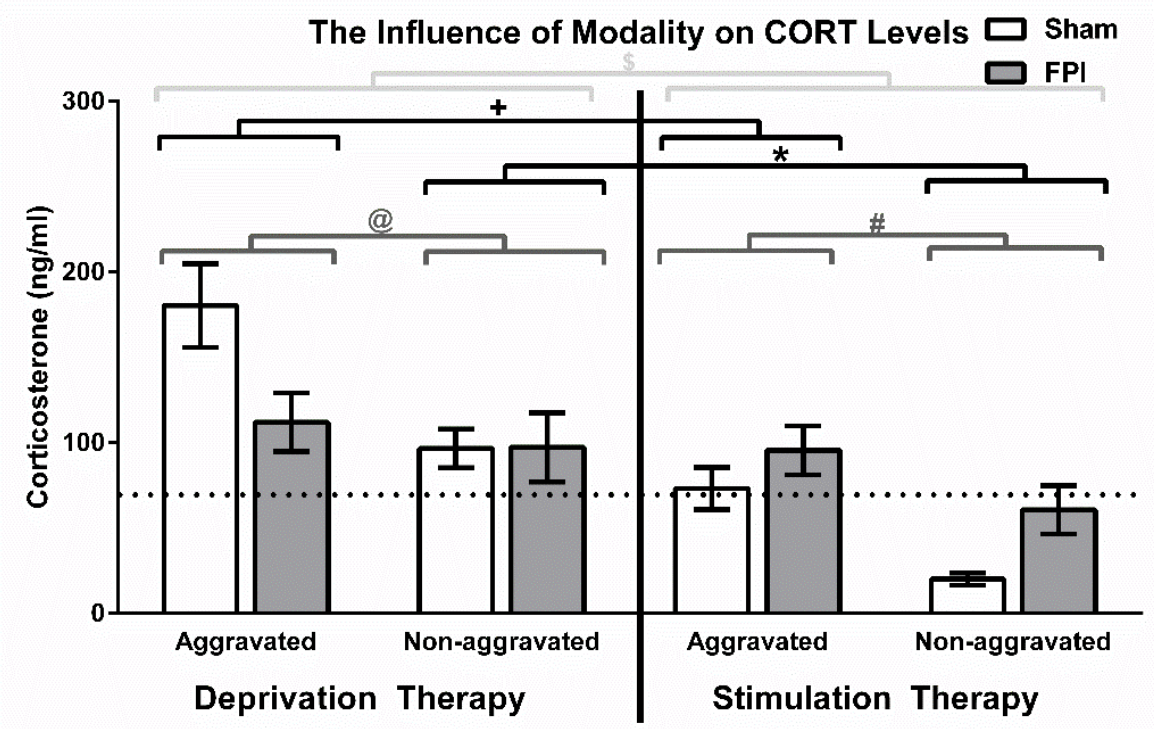

Figure 5. Does modality of rehabilitation influence overall CORT levels? Aggravated and nonaggravated CORT levels for week one and two of each therapy were combined and assessed as a function of therapy modality. Overall, deprivation therapy resulted in greater CORT levels than stimulation therapy (\$, light gray brackets). Both aggravated and non-aggravated CORT levels of deprivation therapy treated rats were elevated in comparison to aggravated and non-aggravated CORT levels of stimulation therapy rats, respectively (+,*; black brackets). Further, aggravated levels of deprivation therapy (@, dark gray brackets) and stimulation therapy (\#, dark gray brackets) were increased in comparison to their respective non-aggravated levels. Bar graphs represent the mean \pm SEM. $\$,+, \#, @,{ }^{*} p<0.05$. Horizontal dotted line represents CORT levels of naive rats $(n=3)$.

\subsection{The effect of deprivation therapy onset on sensory hypersensitivity}

After deprivation therapy at week one or week two post-injury, whisker nuisance scores acquired from sham and FPI rats at 28 days post-injury were compared to establish if timing of deprivation therapy and injury status could alter late-onset sensory 
hypersensitivity to whisker stimulation. A Mann-Whitney unpaired, 2-tailed, nonparametric comparison indicated that there was a significant difference in timing of the deprivation therapy $\left({ }^{\#} U=43.0 ; p<0.05\right.$; figure 4), indicating that deprivation therapy during week two after surgery resulted in greater whisker hypersensitivity than week one post-injury ( $6.83 \pm 0.98$ vs. $2.44 \pm 0.48$, respectively). However, a separate MannWhitney unpaired, 2-tailed, nonparametric comparison failed to find a difference between FPI and sham rats ( $4.42 \pm 0.89$ vs. $5.16 \pm 1.08$, respectively; $U=100.0 ; p=0.58)$. These results indicate that sensory deprivation therapy during week two adversely affected the WNT scores, but not as a function of injury.

\subsection{The effect of deprivation therapy onset on CORT levels in response to the WNT}

To determine the effect of deprivation therapy between week one and two postinjury on sham and FPI rats, aggravated and non-aggravated CORT levels were compared using a mixed ANOVA (figure 6). The results indicated there was no effect of therapy onset $(F(1,25)=0.89, p=0.35)$, injury status $(F(1,25)=3.31, p=0.08)$ or an interaction of therapy onset and injury status $(F(1,25)=3.12, p=0.07)$. However, a within week comparison revealed a main effect between aggravated and non-aggravated CORT levels $(F(1,25)=11.87, p<0.05)$, injury status $(F(1,25)=5.77, p<0.05)$ and significant interactions of CORT levels by week of therapy $(F(1,24)=38.33, p<0.05)$, but not an interaction between therapy onset and injury status $(F(1,24)=0.37, p=0.54)$. These results show that, non-aggravated CORT levels were significantly higher than aggravated levels when deprivation therapy was administered during week one, regardless of injury status $\left(135.79 \pm 21.37>88.41 \pm 24.52, \mathrm{ng} / \mathrm{ml}^{+}\right.$figure 6$)$. When deprivation therapy was administered during week two, aggravated CORT levels were higher than non-aggravated levels $(59.72 \pm 9.33<181.24 \pm 19.25 \mathrm{ng} / \mathrm{ml}$, *figure 6), regardless of injury status. Deprivation during week 2 had a differential effect on FPI and sham rats, with FPI rats having a significantly lower aggravated response in comparison to sham $(148.19 \pm 18.21<236.38 \pm 23.51 \mathrm{ng} / \mathrm{ml}$ figure 6$)$.

Planned comparisons were run to compare individual group differences within each week of deprivation therapy and across weeks using Bonferroni's correction method. The FPI rats with deprivation therapy during week one had significantly higher 
non-aggravated CORT levels compared to the non-aggravated FPI rats with therapy during week two (151.54 \pm 31.87 vs. $44.59 \pm 11.77 \mathrm{ng} / \mathrm{ml} ; t(50)=3.93, p<0.05$, figure 6$)$, indicating that non-aggravated CORT levels were impacted by deprivation therapy onset. Sham and FPI rats with deprivation therapy during week two had higher aggravated CORT levels in comparison to non-aggravated levels (sham-236.38 \pm 14.71 vs. $84.95 \pm 10.35 \mathrm{ng} / \mathrm{ml} ;{ }^{\$}(50)=4.58, p<0.05 ; \mathrm{FPI}-148.19 \pm 24.34$ vs. $44.59 \pm 11.77 \mathrm{ng} / \mathrm{ml}$; $\$ t(50)=4.04 ; p<0.05$; figure 6), however, aggravated levels in shams were significantly greater than aggravated levels in $F P I$ rats $(F(1,14)=9.03, p<0.05)$. Overall, deprivation therapy during week one resulted in increased non-aggravated CORT levels in FPI rats in comparison to their aggravated levels, indicating that the WNT did not elicit a stress response. However, when the deprivation therapy was provided during week two, the non-aggravated CORT levels were lower than aggravated levels, indicating that both sham and FPI rats had a robust stress response to the WNT. Onset of deprivation therapy at week two post-injury differentially influenced aggravated levels of CORT, with FPI rats having significantly lower aggravated CORT levels in comparison with sham aggravated CORT levels. 


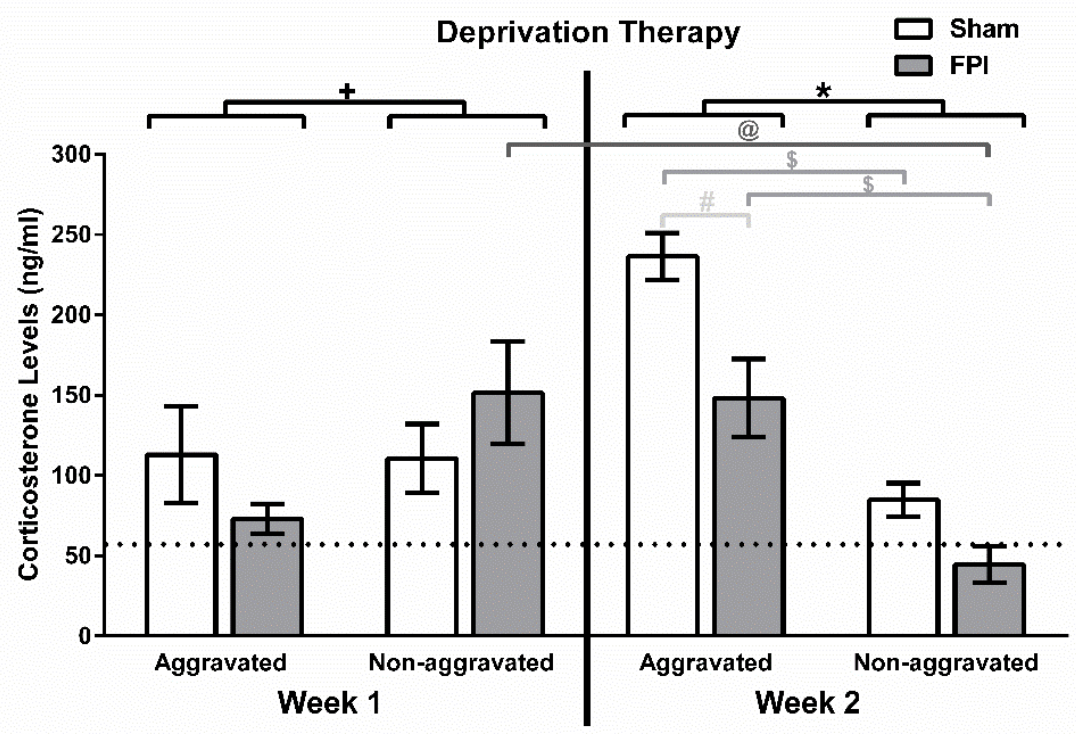

Figure 6. Does onset of deprivation therapy influence CORT levels? Aggravated and non-aggravated CORT levels were evaluated as a function of deprivation therapy onset (week one vs. week 2) and injury status. When sham and FPI rats CORT levels were combined, deprivation therapy during week one postinjury resulted in non-aggravated CORT levels being overall greater than aggravated levels $(+$, black brackets). In addition, there was no CORT response to the WNT at 28 days post-injury. However, deprivation therapy at week two resulted in a robust CORT response to the WNT, with aggravated levels being significantly greater than non-aggravated (*, black brackets). Week one non-aggravated CORT levels in FPI rats were greater than week two non-aggravated CORT levels (@, dark gray line). When deprivation therapy was provided during week two, the non-aggravated CORT levels were lower, but both sham and FPI rats had a robust stress response to the WNT (\$, light gray). Within subjects analysis of week two indicates that aggravated CORT levels in shams were significantly greater than aggravated levels in FPI rats (\#, lightest gray). Bar graphs represent the mean \pm SEM. $\$,+, @,{ }^{*} p<0.05$. Horizontal dotted line represents CORT levels of naive rats $(n=3)$.

\subsection{The effect of stimulation therapy onset on sensory hypersensitivity} After stimulation therapy at one week or two weeks post-injury, WNT scores

acquired from sham and FPI rats at 28 days post-injury were compared to establish if timing of stimulation therapy and injury status could alter late-onset sensory hypersensitivity (figure 4). A Mann-Whitney unpaired, 2-tailed, nonparametric comparison indicated neither stimulation therapy onset $(U=115.0 ; p=0.58)$ nor injury status $(U=106.5 ; p=0.55)$ resulted in altered WNT scores. FPI rats stimulated during week one or week two post-injury performed similar to shams. The stimulation therapy in the tubes resulted WNT scores at or below naïve controls (dotted line), regardless of therapy onset or injury status. 


\subsection{The effect of stimulation therapy onset on CORT levels in response to the WNT}

To determine the effect of stimulation therapy during week one or two post-injury in sham and FPI rats, aggravated and non-aggravated CORT levels were compared using a mixed ANOVA (figure 7). Between-subjects effects indicated there is no main effect of therapy onset $(F(1,20)=1.03, p=0.32)$, but there was a main effect of injury status $(F(1,20)=15.20, p<0.05)$ and an injury status $x$ therapy onset interaction $(F(1,20)=4.55$, $p<0.05)$ for the between subjects comparisons. These results show that, in general, CORT levels in FPI rats were higher than shams regardless of therapy onset or aggravated status. They also show that stimulation therapy during week one resulted in FPI rats with higher average CORT levels (aggravated + non-aggravated) compared to sham at one month post-injury $(88.64 \pm 9.80>32.21 \pm 10.96 \mathrm{ng} / \mathrm{ml})$. However, therapy during week two indicated similar CORT levels between injured and sham rats $(78.18 \pm 7.30>61.66 \pm 8.95 \mathrm{ng} / \mathrm{ml}$ ) (comparisons not shown in figure 7 ).

The within subject comparisons revealed a main effect of CORT levels $(F(1,20)=11.44, p<0.05)$ and an interaction of CORT levels by week of therapy onset $(F(1,20)=10.54, p<0.05)$, but no change in CORT levels by injury status $(F(1,20)=1.46$, $p=0.24)$ or an interaction $(F(1,20)=1.62, p=0.21)$. These results show that following stimulation therapy during week one, there were no differences between aggravated and non-aggravated CORT levels. However, for stimulation therapy during week two, aggravated CORT levels were significantly higher than non-aggravated levels (113.54 $\pm 14.65 \mathrm{ng} / \mathrm{ml}$ vs. $26.32 \pm 8.07$; *figure 7 ). 
Planned comparisons were run to compare individual group differences within each week of stimulation therapy and across weeks using Bonferroni's correction method. With stimulation therapy during week one, there was a significant increase in non-aggravated levels in the FPI rats compared to the shams $(104.09 \pm 27.36 \mathrm{ng} / \mathrm{ml}>$ $15.00 \pm 1.02 ; t(40)=3.50,{ }^{+} p<0.05$, figure 7). Sham and FPI rats with stimulation therapy during week two demonstrated greater aggravated CORT levels than non-aggravated CORT levels (FPI 122.20 $\pm 18.22>34.16 \pm 9.66 \mathrm{ng} / \mathrm{ml}$; ${ }^{\dagger} t(40)=4.93, p<0.05$; sham 104.85 $\pm 13.19>18.48 \pm 5.89 \mathrm{ng} / \mathrm{ml}$; ${ }^{@} t(40)=3.95, p<0.05$, figure 7$)$. Note that CORT levels did not vary substantially from naïve rats when stimulation therapy onset was at one or two weeks post-injury, despite deprivation therapy resulting in higher levels than naïve. Overall, stimulation therapy resulted in lower CORT levels (aggravated and nonaggravated) in comparison to deprivation rats, with stimulation therapy during week one negating a hypersensitive response to the WNT. Onset of stimulation therapy at week one post-injury differentially influenced non-aggravated levels of CORT, with FPI rats having significantly higher non-aggravated CORT levels in comparison to nonaggravated CORT levels in shams.

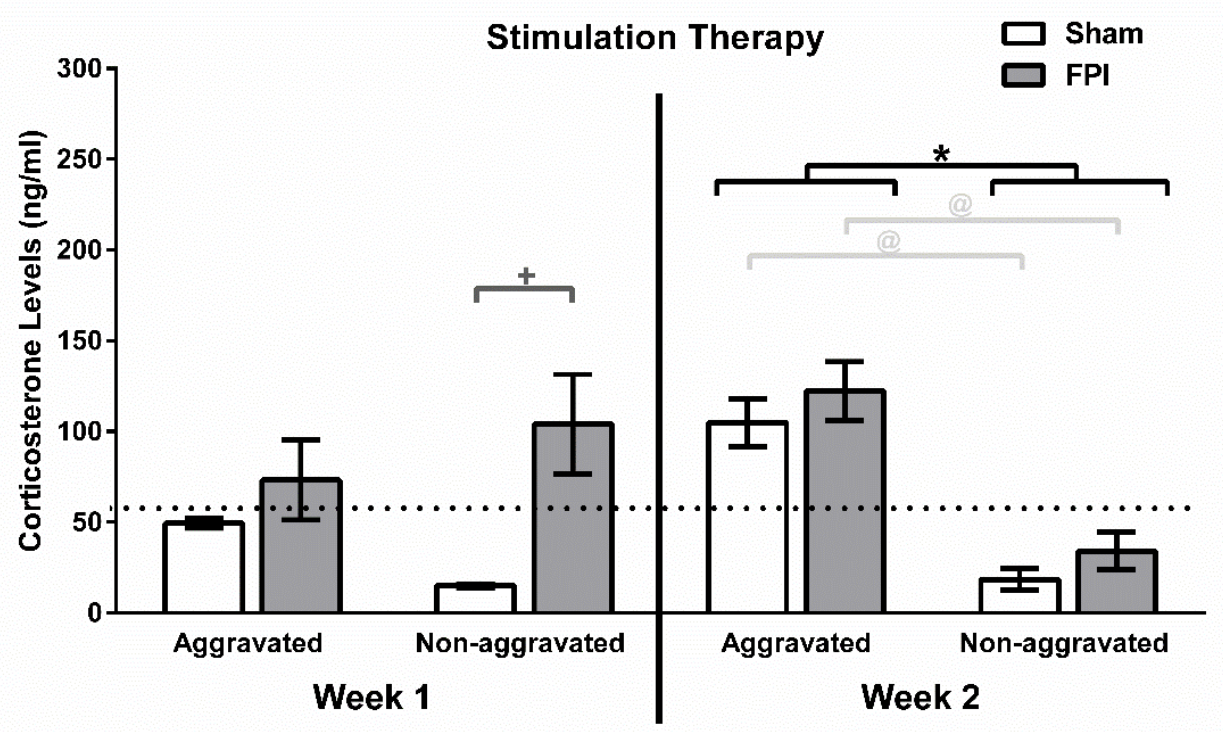

Figure 7. Does onset of stimulation therapy influence CORT levels? Aggravated and non-aggravated CORT levels were evaluated as a function of stimulation therapy onset (week one vs. week two) and injury status. Stimulation therapy during week one post-injury resulted in FPI rats demonstrating a greater nonaggravated CORT level in comparison to sham (+, dark gray brackets) as well as no substantial CORT response to the WNT. When stimulation therapy was administered during week two, aggravated CORT levels were significantly greater than non-aggravated CORT levels, regardless of injury status $\left({ }^{*}\right.$, black brackets; @ light gray brackets). No difference between sham and Bar graphs represent the mean \pm SEM. $+, @,{ }^{\star} p<0.05$. Horizontal dotted line represents CORT levels of naive rats $(n=3)$. 


\section{Discussion}

The present study determined that modality and onset of rehabilitation differentially influenced sensory hypersensitivity and plasma CORT levels in sham and diffuse braininjured rats. Despite no injury-induced changes in behavior, plasma CORT continues to be a relevant marker of stress and aggravation in this paradigm. Deprivation during week two resulted in increased WNT scores and aggravated CORT levels for sham and FPI rats; however, aggravated levels in shams were significantly higher than FPI rats. Stimulation therapy during week one produced significantly higher non-aggravated CORT levels in FPI rats compared to sham. Stimulation therapy during week two resulted in a muted CORT response to the WNT in both sham and injured rats compared to deprivation therapy. Each of these paradigms were capable of changing long-term outcome measures, indicating that rehabilitation approaches can have a lasting impact on both sham and injured rats, with indications that injured rats may respond differently to deprivation therapy during week two and stimulation therapy during week one. These data support that choice of modality and onset of rehabilitation strategies in the clinic may have long-term effects on patients recovering from TBI.

\subsection{Deprivation Therapy}

One of the primary recommendations after a concussion or brain injury is (cognitive) rest and avoidance of high stimulation environments. After TBI, the brain is metabolically challenged, with decreased levels of ATP and compromised mitochondria, where there is evidence that increasing activity would further tax available resources and result in increased pathology and impeded recovery (Ip, Zanier, Moore, Lee, \& Hovda, 2003; Lifshitz et al., 2003). There is also evidence that return to activity too soon can decrease levels of brain-derived neurotrophic factor (BDNF), limiting neuroplasticity and repair, resulting in greater cognitive impairment (Griesbach, Hovda, Molteni, Wu, \& Gomez-Pinilla, 2004). Therefore, deprivation therapy was implemented in this experiment by bilaterally shaving the whiskers to prevent activation of the whisker barrel circuit during week one or week two post-injury while the rat remains in his home cage with cage mate. By shaving the whiskers twice in one week, sensory input would be limited and would not influence the natural repair processes, allowing re-growth of circuits without conflicting and excessive sensory input. 
With deprivation therapy during week one, there was no behavioral or stress response to the WNT at one month post-injury, however sham+injured non-aggravated levels of CORT after week one therapy were elevated in comparison to non-aggravated levels from rats with deprivation therapy during week two (and non-aggravated levels from both weeks of stimulation therapy). So while there was not an adverse behavioral or stress response in sham or injured rats, the duration and underlying mechanisms resulting in elevated non-aggravated CORT levels need further investigation.

Deprivation therapy during week two post-injury resulted in robust behavioral and stress responses to the WNT in sham and injured rats, similar to what we previously reported after 3 weeks of deprivation (McNamara et al., 2010), further supporting that prolonged or late deprivation may not promote recovery. Clinical literature reports longterm rest can result in depression due to removal of normal life circumstances, physical deconditioning and mental health degeneration (DiFazio, Silverberg, Kirkwood, Bernier, \& Iverson, 2016). In adult rats, reduced sensory input through trimmed whiskers causes experience-dependent weakening of synaptic efficacy (Armstrong-James, Diamond, \& Ebner, 1994). If the circuit is weakened, reintroducing large amounts of sensory information in the form of the WNT may overload the circuit resulting in higher WNT scores. Length of the whiskers at the time of the WNT may also influence behavior and stress response, since whiskers have not yet returned to full length $(40-50 \mathrm{~mm})$. The rat's last shave was on day 12 , leaving 16 days for the whiskers to regrow. During this time, it is estimated that the whiskers grow $1 \mathrm{~mm} /$ day, for a total of $\sim 16 \mathrm{~mm}$ by 28 days post-injury (Ibrahim \& Wright, 1975). Further studies would need to confirm that whisker length did not impact response to the WNT.

Deprivation during week two caused aggravated CORT levels in FPI rats to be significantly lower than sham. It is possible that deprivation therapy was stressful to FPI and sham rats, causing a greater CORT response to whisker stimulation and a robust behavioral response in the WNT. The difference between FPI and sham rats may be that the FPI rats are demonstrating a blunted CORT response to the WNT at 28 days post-injury, in line with our previous report that FPI rats have significantly lower baseline CORT levels and a blunted response to restraint stress by 2 months post-injury in comparison to shams (Rowe et al., 2016). Blunted CORT responses to restraint stress 
at 1 month post-injury have also been reported after moderate severity controlled cortical impact-induced focal injury (Taylor et al, 2007). If deprivation therapy is an added stressor to sham animals, it is possible that deprivation in addition to the TBI is enough to accelerate changes that we see in untreated FPI rats.

High non-aggravated CORT levels, indicated after deprivation and stimulation during week one post-injury, may be indicative of chronically elevated CORT levels. Chronically elevated CORT levels can reduce long-term potentiation, promote long-term depression and impair cognitive function (de Quervain, Roozendaal, \& McGaugh, 1998; Foy, Stanton, Levine, \& Thompson, 1987; Kim, Song, \& Kosten, 2006; Pavlides, Kimura, Magarinos, \& McEwen, 1995). Notably, rats treated with chronic CORT for 3 months after TBI had worse behavioral outcomes (White-Gbadebo \& Hamm, 1993). Future experiments are required to test how long elevated CORT levels persist, the impact on the hypothalamic-pituitary-adrenal axis, and the influence on affective and cognitive behaviors.

In humans and rats, repeated exposure to stress hormones (an 'aggravated' response) can impact brain plasticity, regardless of injury status (Kaplan et al., 2010) (de la Tremblaye et al., 2017; Gomez-Pinilla, 2008; Griesbach et al., 2014; Schaaf et al., 2000; White-Gbadebo \& Hamm, 1993). Upregulation of BDNF after TBI can contribute to synaptic plasticity, learning and memory, and improving behavioral outcomes in brain injured rats (Gomez-Pinilla, 2008). However, activation of the stress response (elevated circulating levels of CORT) can downregulate BDNF, thereby prohibiting positive benefits, which could be more detrimental for injured rats (Schaaf et al., 2000). Therefore, when evaluating the effectiveness of rehabilitation, it is important to minimize stressful situations. Further experiments are required to test whether deprivation therapy would compromise BDNF levels and cognitive testing to determine the full extent on neuroplasticity.

\subsection{Stimulation Therapy}

Circuit-directed sensory rehabilitation was compared in a recent review on the influence of stimulation parameters in a clinical setting. They reported that methods like transcranial magnetic stimulation, transcutaneous electrical stimulation, single and 
multiple bursts stimulation protocols were capable of inducing Hebbian type synaptic mechanisms in turn stimulating adaptive reorganization where maladaptive reorganization has resulted in sensory deficits(Parianen Lesemann, Reuter, \& Godde, 2015). Despite variations in protocols that limited direct comparisons, repeated exposure to stimulation and duration of exposure longer than two hours were beneficial to patients. This supports a repeated paradigm for rodent rehabilitation paradigms. Further, 15 minute bouts of stimulation in adult rodents should translate into greater than two hours of stimulation in humans based on 1 human year being equivalent to 10.5-11.8 days in a young adult rat (Sengupta, 2013).

Based, in part, on previous experimental studies on enriched environment (EE) intervention, the tube stimulation intervention was introduced to provide purposeful exploration of unfamiliar tunnels that would require activation of the whisker barrel circuit to navigate. The tubes were designed to be a whisker-specific exploration task rather than a learning task, which would require organized re-growth of circuits and thus reduce the sensory hypersensitivity. With meaningful exploration using the whiskers, neuroplastic recovery of circuits would be encouraged. Tube therapy stimulated the somatosensory region of the rat's brain, as demonstrated by time-dependent activation of activity-regulated cytoskeletal (ARC) genes. Previously, we demonstrated that BDNF and ARC genes, both implicated in synaptic plasticity, were time-dependently elevated in the somatosensory cortex after manual and exploratory whisker stimulation, indicating that stimulation therapy is capable of promoting plasticity (El-Sayed, HofmanBang, \& Mikkelsen, 2011; Khodadad et al., 2015; Leal, Comprido, \& Duarte, 2014; Li et al., 2015; Tan et al., 2007).

Because a single bout or continuous EE is not consistent with strategies used in human rehabilitation, these studies took rats out of their normal environment and exposed them to 15 minutes bouts of circuit-directed stimulation three times in a week to simulate aspects of out-patient rehabilitation that we had previously tested in our laboratory (McNamara et al., 2010; de Witt et al., 2011; Tan et al., 2007). We developed a strategy for tube therapy trials performed eight times in one session and repeated alternately on three days in one week to replicate treatment from a physical therapy outpatient model. Low WNT scores indicate that this method may be successful in 
decreasing late-onset sensory hypersensitivity and lowering CORT levels in comparison to deprivation therapy. However, increased non-aggravated CORT levels in FPI rats after stimulation therapy during week one post-injury in comparison to shams indicates that early stimulation, while better than deprivation, may not be optimal in injured rats. The intensity and duration of rehabilitation therapies required to best attenuate injuryinduced deficits as well as maintain the long-term benefits of rehabilitation remains to be determined. With deprivation during week two and stimulation during week one differentially influencing CORT levels, future studies should also consider a combinatorial approach that starts with early rest (deprivation week one) followed by stimulation during week two.

WNT scores and CORT levels from naïve rats in this study were similar to shams from previous studies that were not exposed to rehabilitation. Previous measurements of late-onset sensory hypersensitivity to whisker stimulation using the WNT indicated that average sham scores range from $1.7 \pm 0.2$ through $4.0 \pm 0.5$ while FPI scores range from 6.5 \pm 0.6 through 9.5 \pm 0.5 (mean $\pm S E M$; Learoyd \& Lifshitz, 2012; McNamara et al., 2010; Thomas et al., 2012). Naïve animals in this study had an average score of 2.27. Deprivation during week two resulted in scores that were similar to FPI rats from previous studies, regardless of injury status, with week one deprivation and week one and two stimulation scores remaining within sham range. Previously published aggravated and non-aggravated CORT levels in shams $(74 \pm 12 \mathrm{ng} / \mathrm{ml})$ were also similar to naïve rats in this study $(60 \pm 20 \mathrm{ng} / \mathrm{ml})$. Aggravated levels of CORT in FPI rats without exposure to rehabilitation was approximately $125 \pm 18 \mathrm{ng} / \mathrm{ml}$ (McNamara et al., 2012), also within range of aggravated CORT levels in this study, with the exception of sham animals with deprivation therapy during week two, where aggravated levels were $\sim 235$ $\mathrm{ng} / \mathrm{ml}$. These reference data are reported to help put the results into context with regard to efficacy, but studies designed for a direct comparison are necessary.

While our laboratory and others have repeatedly published the development of late-onset sensory hypersensitivity to whisker stimulation, we did not find differences between injured and sham rats in this study (Alwis, Yan, Morganti-Kossmann, \& Rajan, 2012; Lafrenaye, Krahe, \& Povlishock, 2014; Learoyd \& Lifshitz, 2012; McNamara et al., 2010; Thomas et al., 2012). In this set of experiments, it is possible that repeated 
handling of the rats and rehabilitation in both injured and sham rats muted the normal behavioral response to the WNT in injured rats. Repeated gentle handling of rats is capable of decreasing anxiety-like behavior, CORT levels and improve cognitive skills in Sprague-Dawley rats in comparison to non-handled controls (Costa, Tamascia, Nogueira, Casarini, \& Marcondes, 2012; Deutsch-Feldman, Picetti, Seip-Cammack, Zhou, \& Kreek, 2015). The premise of rehabilitation is to restore function by strengthening remaining connections, rerouting maladapted connections and promoting compensation for improved general function. However, tube stimulation in intact sham rats likely serves as a form of training that is capable of strengthening intact circuitry (Khodadad et al., 2015), and it's equally likely to benefit sham as it does injured rats. Now that we have identified therapeutic approaches that differentially influence outcome measures, future studies will continue to optimize timing and duration to provide further insight as to the efficacy of the rehabilitation strategies on persisting impairment. Also, since we only used a single 'intensity' of stimulation in these experiments, quantifiable therapeutic biomarkers, like ARC, hold potential for determining optimal levels of stimulation that promote recovery, without adverse effects (Khodadad et al., 2015; Lifshitz et al., 2016).

\subsection{Conclusion}

Onset of intervention may be pivotal in recovery, and at present, is not universally agreed upon in a clinical setting. Discharge papers outlining early rest with gradual return to usual activity did not significantly impact TBI symptoms at two weeks or one month following mild TBI in comparison to nondescript discharge papers in patients (Varner et al., 2017). Deprivation or active rehabilitation is typically initiated as early as possible, though experimental studies show that intervention initiated during week one following injury may have a negative impact on neuroplasticity (Griesbach, Gomez-Pinilla, et al., 2004). Our results demonstrate that tube therapy is capable of activating the whisker circuit and that modality and onset of rehabilitation can differentially influence lasting behavior and stress responses between injured and sham rats. Stimulation therapy resulted in overall lower CORT levels, suggesting it may be a better approach than deprivation therapy in this regard. 
Overall, these data indicate that early therapeutic interventions can have a lasting influence on behavioral recovery of sensory circuits following injury, though preferential timing for intervention initiation is still unclear. Further exploration of the whisker barrel circuit may guide in understanding functional recovery of sensorimotor systems and provide an evidence based model for clinical intervention.

\section{Acknowledgements}

The authors would like to thank Kelley Hall and Amanda Lisembee for technical support and Dr. Cristina Morganti-Kossmann for valuable feedback on the manuscript. These data were collected at the Spinal Cord and Brain Injury Research Center at the University of Kentucky. Funding provided by KSCHIRT \#7-11, NIH R03 NS07709801A1, NIH NINDS R01 NS065052, 1 P30 NS051220-01; Arizona Biomedical Research Commission through the Arizona Department of Health Services (ADHS14-00003606) and Phoenix Children's Hospital Mission Support Funds. 


\section{References}

Alwis, D. S., Yan, E. B., Morganti-Kossmann, M. C., \& Rajan, R. (2012). Sensory cortex underpinnings of traumatic brain injury deficits. PLoS One, 7(12), e52169.

Armstrong-James, M., Diamond, M. E., \& Ebner, F. F. (1994). An innocuous bias in whisker use in adult rats modifies receptive fields of barrel cortex neurons. Journal of Neuroscience, 14(11 Pt 2), 6978-6991.

Cao, T., Thomas, T. C., Ziebell, J. M., Pauly, J. R., \& Lifshitz, J. (2012). Morphological and genetic activation of microglia after diffuse traumatic brain injury in the rat. Neuroscience, 225, 65-75.

Ciricillo, S. P., Hill, M. P., Gonzalez, M. F., Smalley, S., Morton, M. T., \& Sharp, F. R. (1994). Whisker stimulation metabolically activates thalamus following cortical transplantation but not following cortical ablation. Neuroscience, 59(4), 975-992.

Gerberding, J.L. (2003). National Center for Injury Prevention and Control. Report to Congress on Mild Traumatic Brain Injury in the United States: Steps to Prevent a Serious Public Health Problem. Atlanta, GA: Centers for Disease Control and Prevention. http://www.cdc.gov/traumaticbraininjury/pdf/mtbireport-a.pdf.

Coronado, V. G., Xu, L., Basavaraju, S. V., McGuire, L. C., Wald, M. M., Faul, M. D., . . . Hemphill, J. D. (2011). Surveillance for traumatic brain injury-related deaths-United States, 1997-2007. Morbidity and Mortality Weekly Report Surrveillance Summaries, 60(5), 1-32.

Costa, R., Tamascia, M. L., Nogueira, M. D., Casarini, D. E., \& Marcondes, F. K. (2012). Handling of adolescent rats improves learning and memory and decreases anxiety. Journal of American Association of Laboratory Animal Science, 51(5), 548-553.

de la Tremblaye, P. B., O'Neil, D. A., LaPorte, M. J., Cheng, J. P., Beitchman, J. A., Thomas, T. C., . . . Kline, A. E. (2017). Elucidating opportunities and pitfalls in the treatment of experimental traumatic brain injury to optimize and facilitate clinical translation. Neuroscience \& Biobehavioral Reviews.

de Quervain, D. J., Roozendaal, B., \& McGaugh, J. L. (1998). Stress and glucocorticoids impair retrieval of long-term spatial memory. Nature, 394(6695), 787-790.

de Witt, B. W., Ehrenberg, K. M., McAloon, R. L., Panos, A. H., Shaw, K. E., Raghavan, P. V., . . Kline, A. E. (2011). Abbreviated environmental enrichment enhances neurobehavioral recovery comparably to continuous exposure after traumatic brain injury. Neurorehabilitation \& Neural Repair, 25(4), 343-350.

Deutsch-Feldman, M., Picetti, R., Seip-Cammack, K., Zhou, Y., \& Kreek, M. J. (2015). Effects of handling and vehicle injections on adrenocorticotropic and corticosterone concentrations in Sprague-Dawley compared with Lewis rats. ournal of American Association of Laboratory Animal Science, 54(1), 35-39.

DiFazio, M., Silverberg, N. D., Kirkwood, M. W., Bernier, R., \& Iverson, G. L. (2016). Prolonged Activity Restriction After Concussion: Are We Worsening Outcomes? Clinical Pediatrics (Phila), 55(5), 443-451. 
Eastman, A., \& Chang, D. G. (2015). Return to Learn: A review of cognitive rest versus rehabilitation after sports concussion. NeuroRehabilitation, 37(2), 235-244.

El-Sayed, M., Hofman-Bang, J., \& Mikkelsen, J. D. (2011). Effect of brain-derived neurotrophic factor on activity-regulated cytoskeleton-associated protein gene expression in primary frontal cortical neurons. Comparison with NMDA and AMPA. European journal of pharmacology, 660(2-3), 351-357.

Fenn, A. M., Skendelas, J. P., Moussa, D. N., Muccigrosso, M. M., Popovich, P. G., Lifshitz, J., . . . Godbout, J. P. (2015). Methylene blue attenuates traumatic brain injury-associated neuroinflammation and acute depressive-like behavior in mice. Journal of Neurotrauma, 32(2), 127-138.

Foy, M. R., Stanton, M. E., Levine, S., \& Thompson, R. F. (1987). Behavioral stress impairs long-term potentiation in rodent hippocampus. Behav Neural Biol, 48(1), 138-149.

Gomez-Pinilla, F. (2008). The influences of diet and exercise on mental health through hormesis. Ageing Res Rev, 7(1), 49-62.

Gomez-Pinilla, F., Ying, Z., Agoncillo, T., \& Frostig, R. (2011). The influence of naturalistic experience on plasticity markers in somatosensory cortex and hippocampus: effects of whisker use. Brain Research, 1388, 39-47.

Griesbach, G. S., Gomez-Pinilla, F., \& Hovda, D. A. (2004). The upregulation of plasticity-related proteins following TBI is disrupted with acute voluntary exercise. Brain Research, 1016(2), 154-162.

Griesbach, G. S., Gomez-Pinilla, F., \& Hovda, D. A. (2007). Time window for voluntary exercise-induced increases in hippocampal neuroplasticity molecules after traumatic brain injury is severity dependent. Journal Neurotrauma, 24(7), 11611171.

Griesbach, G. S., Hovda, D. A., Molteni, R., Wu, A., \& Gomez-Pinilla, F. (2004). Voluntary exercise following traumatic brain injury: Brain-derived neurotrophic factor upregulation and recovery of function. Neuroscience, 125(1), 129-139.

Griesbach, G. S., Hovda, D. A., Tio, D. L., \& Taylor, A. N. (2011). Heightening of the stress response during the first weeks after a mild traumatic brain injury. Neuroscience, 178, 147-158.

Griesbach, G. S., Tio, D. L., Nair, S., \& Hovda, D. A. (2014). Recovery of stress response coincides with responsiveness to voluntary exercise after traumatic brain injury. Journal of Neurotrauma, 31(7), 674-682.

Griesbach, G. S., Tio, D. L., Vincelli, J., McArthur, D. L., \& Taylor, A. N. (2012). Differential effects of voluntary and forced exercise on stress responses after traumatic brain injury. Journal of Neurotrauma, 29(7), 1426-1433.

Grossman, E. J., Inglese, M., \& Bammer, R. (2010). Mild traumatic brain injury: is diffusion imaging ready for primetime in forensic medicine? Topics on Magnetic Resonance Imaging, 21(6), 379-386. 
Guimaraes, J. S., Freire, M. A., Lima, R. R., Souza-Rodrigues, R. D., Costa, A. M., dos Santos, C. D., . . Gomes-Leal, W. (2009). [Mechanisms of secondary degeneration in the central nervous system during acute neural disorders and white matter damage]. Revue Neurologique, 48(6), 304-310.

Hall, K. D., \& Lifshitz, J. (2010). Diffuse traumatic brain injury initially attenuates and later expands activation of the rat somatosensory whisker circuit concomitant with neuroplastic responses. Brain Research, 1323, 161-173.

Harrison, J. L., Rowe, R. K., Ellis, T. W., Yee, N. S., O'Hara, B. F., Adelson, P. D., \& Lifshitz, J. (2015). Resolvins AT-D1 and E1 differentially impact functional outcome, post-traumatic sleep, and microglial activation following diffuse brain injury in the mouse. Brain, Behavior, and Immunity. 47, 131-140.

Hoffman, A. N., Paode, P. R., May, H. G., Ortiz, J. B., Kemmou, S., Lifshitz, J., . . . Thomas, T. C. (2016). Early and Persistent Dendritic Hypertrophy in the Basolateral Amygdala Following Experimental Diffuse Traumatic Brain Injury. Journal of Neurotrauma.

Hosseini, A. H., \& Lifshitz, J. (2009). Brain injury forces of moderate magnitude elicit the fencing response. Medicine \& Science in Sports \& Exercise, 41(9), 1687-1697.

Ibrahim, L., \& Wright, E. A. (1975). The growth of rats and mice vibrissae under normal and some abnormal conditions. Journal of Embryology and Experimental Morphology, 33(4), 831-844.

Ip, E. Y., Zanier, E. R., Moore, A. H., Lee, S. M., \& Hovda, D. A. (2003). Metabolic, neurochemical, and histologic responses to vibrissa motor cortex stimulation after traumatic brain injury. Journal of Cerebral Blood Flow \& Metabolism, 23(8), 900-910.

Johansson, K., \& Arvidsson, J. (1994). Central plasticity in rat trigeminal primary sensory neurons innervating vibrissae after neonatal peripheral nerve injury. Neuroscience Letters, 167(1-2), 187-190.

Kaplan, G. B., Vasterling, J. J., \& Vedak, P. C. (2010). Brain-derived neurotrophic factor in traumatic brain injury, post-traumatic stress disorder, and their comorbid conditions: role in pathogenesis and treatment. Behavioural Pharmacology, 21(56), 427-437.

Khodadad, A., Adelson, P. D., Lifshitz, J., \& Thomas, T. C. (2015). The time course of activity-regulated cytoskeletal (ARC) gene and protein expression in the whiskerbarrel circuit using two paradigms of whisker stimulation. Behavioural Brain Research, 284, 249-256.

Kim, J. J., Song, E. Y., \& Kosten, T. A. (2006). Stress effects in the hippocampus: synaptic plasticity and memory. Stress, 9(1), 1-11.

Lafrenaye, A. D., Krahe, T. E., \& Povlishock, J. T. (2014). Moderately elevated intracranial pressure after diffuse traumatic brain injury is associated with exacerbated neuronal pathology and behavioral morbidity in the rat. Journal of cerebral blood flow and metabolism : official journal of the International Society of Cerebral Blood Flow and Metabolism, 34(10), 1628-1636. 
Leal, G., Comprido, D., \& Duarte, C. B. (2014). BDNF-induced local protein synthesis and synaptic plasticity. Neuropharmacology, 76 Pt C, 639-656.

Learoyd, A. E., \& Lifshitz, J. (2012). Comparison of rat sensory behavioral tasks to detect somatosensory morbidity after diffuse brain-injury. Behavioural Brain Research, 226(1), 197-204.

Lew, H. L., Pogoda, T. K., Baker, E., Stolzmann, K. L., Meterko, M., Cifu, D. X., . . . Hendricks, A. M. (2011). Prevalence of dual sensory impairment and its association with traumatic brain injury and blast exposure in OEF/OIF veterans. $J$ Head Trauma Rehabilitation, 26(6), 489-496.

Li, Y., Pehrson, A. L., Waller, J. A., Dale, E., Sanchez, C., \& Gulinello, M. (2015). A critical evaluation of the activity-regulated cytoskeleton-associated protein (Arc/Arg3.1)'s putative role in regulating dendritic plasticity, cognitive processes, and mood in animal models of depression. Frontiers in Neuroscience, 9, 279.

Lifshitz, J., Friberg, H., Neumar, R. W., Raghupathi, R., Welsh, F. A., Janmey, P., . . . McIntosh, T. K. (2003). Structural and functional damage sustained by mitochondria after traumatic brain injury in the rat: evidence for differentially sensitive populations in the cortex and hippocampus. Journal of Cerebral Blood Flow and Metabolism , 23(2), 219-231.

Lifshitz, J., Kelley, B. J., \& Povlishock, J. T. (2007). Perisomatic thalamic axotomy after diffuse traumatic brain injury is associated with atrophy rather than cell death. Journal of Neuropathology \& Eperimental Neurology, 66(3), 218-229.

Lifshitz, J., \& Lisembee, A. M. (2012). Neurodegeneration in the somatosensory cortex after experimental diffuse brain injury. Brain Structure \& Function, 217(1), 49-61.

Lifshitz, J., Rowe, R. K., Griffiths, D. R., Evilsizor, M. N., Thomas, T. C., Adelson, P. D., \& McIntosh, T. K. (2016). Clinical relevance of midline fluid percussion brain injury: Acute deficits, chronic morbidities and the utility of biomarkers. Brain Injury, 1-9.

Lima, F. P., Lima, M. O., Leon, D., Lucareli, P. R., Falcon, C., Cogo, J. C., . . Junque, C. (2011). fMRI of the sensorimotor cortex in patients with traumatic brain injury after intensive rehabilitation. Neurological Sciences, 32(4), 633-639.

Livak, K. J., \& Schmittgen, T. D. (2001). Analysis of relative gene expression data using real-time quantitative PCR and the 2(-Delta Delta C(T)) Method. Methods, 25(4), 402-408.

Lyford, G. L., Yamagata, K., Kaufmann, W. E., Barnes, C. A., Sanders, L. K., Copeland, N. G., . . . Worley, P. F. (1995). Arc, a growth factor and activity-regulated gene, encodes a novel cytoskeleton-associated protein that is enriched in neuronal dendrites. Neuron, 14(2), 433-445.

Masel, B. E., \& DeWitt, D. S. Traumatic brain injury: a disease process, not an event. J Neurotrauma, 27(8), 1529-1540.

Matter, A. M., Folweiler, K. A., Curatolo, L. M., \& Kline, A. E. (2011). Temporal effects of environmental enrichment-mediated functional improvement after experimental 
traumatic brain injury in rats. Neurorehabilitation \& Neural Repair, 25(6), 558564.

McNamara, K. C., Lisembee, A. M., \& Lifshitz, J. (2010). The whisker nuisance task identifies a late-onset, persistent sensory sensitivity in diffuse brain-injured rats. Journal of Neurotrauma, 27(4), 695-706.

Nalder, E., Fleming, J., Foster, M., Cornwell, P., Shields, C., \& Khan, A. (2011). Identifying Factors Associated With Perceived Success in the Transition From Hospital to Home After Brain Injury. Journal of Head Trauma Rehabilitation.

Niechwiej-Szwedo, E., Inness, E. L., Howe, J. A., Jaglal, S., Mcllroy, W. E., \& Verrier, M. C. (2007). Changes in gait variability during different challenges to mobility in patients with traumatic brain injury. Gait Posture, 25(1), 70-77.

O'Connor, W. T., Smyth, A., \& Gilchrist, M. D. (2011). Animal models of traumatic brain injury: a critical evaluation. Pharmacology \& Therapeutics, 130(2), 106-113.

Parianen Lesemann, F. H., Reuter, E. M., \& Godde, B. (2015). Tactile stimulation interventions: influence of stimulation parameters on sensorimotor behavior and neurophysiological correlates in healthy and clinical samples. Neuroscience \& Biobehavioral Reviews, 51, 126-137.

Pavlides, C., Kimura, A., Magarinos, A. M., \& McEwen, B. S. (1995). Hippocampal homosynaptic long-term depression/depotentiation induced by adrenal steroids. Neuroscience, 68(2), 379-385.

Ponsford, J., Cameron, P., Fitzgerald, M., Grant, M., \& Mikocka-Walus, A. (2011). Longterm outcomes after uncomplicated mild traumatic brain injury: a comparison with trauma controls. Journal of Neurotrauma, 28(6), 937-946.

Rinne, M. B., Pasanen, M. E., Vartiainen, M. V., Lehto, T. M., Sarajuuri, J. M., \& Alaranta, H. T. (2006). Motor performance in physically well-recovered men with traumatic brain injury. Journal of Rehabilitation Medicine, 38(4), 224-229.

Rowe, R. K., Rumney, B. M., May, H. G., Permana, P., Adelson, P. D., Harman, S. M., . . . Thomas, T. (2016). Diffuse traumatic brain injury affects chronic corticosterone function in the rat. Endocrine Connectections, 5(4), 152-166.

Saavedra, J. M., Sanchez-Lemus, E., \& Benicky, J. (2011). Blockade of brain angiotensin II AT1 receptors ameliorates stress, anxiety, brain inflammation and ischemia: Therapeutic implications. Psychoneuroendocrinology, 36(1), 1-18.

Sanders, M. J., Dietrich, W. D., \& Green, E. J. (2001). Behavioral, electrophysiological, and histopathological consequences of mild fluid-percussion injury in the rat. Brain Research, 904(1), 141-144.

Sawyer, Q., Vesci, B., \& McLeod, T. C. (2016). Physical Activity and Intermittent Postconcussion Symptoms After a Period of Symptom-Limited Physical and Cognitive Rest. Journal of Athletic Training, 51(9), 739-742.

Schaaf, M. J., De Kloet, E. R., \& Vreugdenhil, E. (2000). Corticosterone effects on BDNF expression in the hippocampus. Implications for memory formation. Stress, 3(3), 201-208. 
Sengupta, P. (2013). The Laboratory Rat: Relating Its Age With Human's. International Journal of Preventative Medicine, 4(6), 624-630.

Sheedy, J., Geffen, G., Donnelly, J., \& Faux, S. (2006). Emergency department assessment of mild traumatic brain injury and prediction of post-concussion symptoms at one month post injury. Journal of Clinical and Experimental Neuropsychology, 28(5), 755-772.

Sosnoff, J. J., Broglio, S. P., Shin, S., \& Ferrara, M. S. (2011). Previous mild traumatic brain injury and postural-control dynamics. Journal of Athletic Training, 46(1), 8591.

Sozda, C. N., Hoffman, A. N., Olsen, A. S., Cheng, J. P., Zafonte, R. D., \& Kline, A. E. (2010). Empirical comparison of typical and atypical environmental enrichment paradigms on functional and histological outcome after experimental traumatic brain injury. Journal of Neurotrauma, 27(6), 1047-1057.

Tan, J., Ruttiger, L., Panford-Walsh, R., Singer, W., Schulze, H., Kilian, S. B., . . . Knipper, M. (2007). Tinnitus behavior and hearing function correlate with the reciprocal expression patterns of BDNF and Arg3.1/arc in auditory neurons following acoustic trauma. Neuroscience, 145(2), 715-726.

Tapper, A., Gonzalez, D., Roy, E., \& Niechwiej-Szwedo, E. (2016). Executive function deficits in team sport athletes with a history of concussion revealed by a visualauditory dual task paradigm. Journal of Sports Science, 1-10.

Thomas, T. C., Hinzman, J. M., Gerhardt, G. A., \& Lifshitz, J. (2012). Hypersensitive glutamate signaling correlates with the development of late-onset behavioral morbidity in diffuse brain-injured circuitry. Journal of Neurotrauma, 29(2), 187200.

Thomas, T. C., Ogle, S. B., Rumney, B. M., May, H. G., Adelson, P. D., \& Lifshitz, J. (2016). Does time heal all wounds? Experimental diffuse traumatic brain injury results in persisting histopathology in the thalamus. Behavioural Brain Research.

Thompson, H. J., Lifshitz, J., Marklund, N., Grady, M. S., Graham, D. I., Hovda, D. A., \& McIntosh, T. K. (2005). Lateral fluid percussion brain injury: a 15-year review and evaluation. Journal of Neurotrauma, 22(1), 42-75.

Varner, C. E., McLeod, S., Nahiddi, N., Lougheed, R. E., Dear, T. E., \& Borgundvaag, B. (2017). Cognitive Rest and Graduated Return to Usual Activities Versus Usual Care for Mild Traumatic Brain Injury: A Randomized Controlled Trial of Emergency Department Discharge Instructions. Academic Emergency Medicine, 24(1), 75-82.

White-Gbadebo, D., \& Hamm, R. J. (1993). Chronic corticosterone treatment potentiates deficits following traumatic brain injury in rats: implications for aging. Journal of Neurotrauma, 10(3), 297-306.

Wilson, D. J., Powell, M., Gorham, J. L., \& Childers, M. K. (2006). Ambulation training with and without partial weightbearing after traumatic brain injury: results of a randomized, controlled trial. American Journal of Physical Medicine \& Rehabilitation, 85(1), 68-74. 
Witgen, B. M., Lifshitz, J., Smith, M. L., Schwarzbach, E., Liang, S. L., Grady, M. S., \& Cohen, A. S. (2005). Regional hippocampal alteration associated with cognitive deficit following experimental brain injury: a systems, network and cellular evaluation. Neuroscience, 133(1), 1-15.

Wogensen, E., Mala, H., \& Mogensen, J. (2015). The Effects of Exercise on Cognitive Recovery after Acquired Brain Injury in Animal Models: A Systematic Review. Neural Plasticity, 2015, 830871.

Wood, R. L., \& Rutterford, N. A. (2006). Psychosocial adjustment 17 years after severe brain injury. Journal of Neurology, Neurosurgery, and Psychiatry, 77(1), 71-73. 